\title{
MiRNA-145 increases therapeutic sensibility to gemcitabine treatment of pancreatic adenocarcinoma cells
}

\author{
Yong Lin ${ }^{1,2}$, Xin $\mathrm{Ge}^{3,4}$, Yiyang Wen ${ }^{3}$, Zhu-Mei Shí, ${ }^{3,5}$ Qiu-Dan Chen' ${ }^{2}$ Min Wang ${ }^{3}$, \\ Ling-Zhi Liu' ${ }^{6}$, Bing-Hua Jiang ${ }^{3,6}$, Yuan Lu ${ }^{1}$ \\ ${ }^{1}$ Department of Laboratory Medicine, Huashan Hospital of Fudan University, Shanghai, China \\ ${ }^{2}$ The Department of Clinical Laboratory, Central Laboratory, Jing'an District Centre Hospital of Shanghai, Huashan Hospital \\ of Fudan University Jing'An Branch, Shanghai, China \\ ${ }^{3}$ State Key Lab of Reproductive Medicine, Key Laboratory of Human Functional Genomics of Jiangsu Province, Jiangsu Key \\ Laboratory of Cancer Biomarkers, Prevention, and Treatment Department of Pathology, Cancer Center, Nanjing Medical \\ University, Nanjing, China \\ ${ }^{4}$ Ninggao Personalized Medicine and Technology Innovation Center, Nanjing, China \\ ${ }^{5}$ Department of Neurosurgery, The First Affiliated Hospital of Nanjing Medical University, Nanjing, China \\ ${ }^{6}$ Department of Pathology, Anatomy and Cell Biology, Thomas Jefferson University, Philadelphia, PA, United States of America \\ Correspondence to: Bing-Hua Jiang, email: binghjiang@yahoo.com, bhjiang@jefferson.edu \\ Yuan Lu, email: luyuan@sccl.org.cn \\ Keywords: pancreatic adenocarcinoma, gemcitabine, miR-145, p70S6K1, chemoresistance
}

Received: May 09, $2016 \quad$ Accepted: August 25, $2016 \quad$ Published: September 27, 2016

ABSTRACT

\begin{abstract}
Pancreatic adenocarcinoma is one of the most leading causes of cancer-related deaths worldwide. Although recent advances provide various treatment options, pancreatic adenocarcinoma has poor prognosis due to its late diagnosis and ineffective therapeutic multimodality. Gemcitabine is the effective first-line drug in pancreatic adenocarcinoma treatment. However, gemcitabine chemoresistance of pancreatic adenocarcinoma cells has been a major obstacle for limiting its treatment effect. Our study found that p7056K1 plays an important role in gemcitabine chemoresistance. MiR-145 is a tumor suppressor which directly targets p7056K1 for inhibiting its expression in pancreatic adenocarcinoma, providing new therapeutic scheme. Our findings revealed a new mechanism underlying gemcitabine chemoresistance in pancreatic adenocarcinoma cells.
\end{abstract}

\section{INTRODUCTION}

Pancreatic adenocarcinoma is the fourth leading cause of cancer-related death around the world with extremely poor prognosis [1]. The five-year survival rate for pancreatic adenocarcinoma patients is approximately $6 \%$, with a median survival of 4-6 months. Thus, pancreatic adenocarcinoma is often diagnostic after it is well-advanced because of its asymptomatic nature [2]. Less than $15 \%$ pancreatic adenocarcinoma patients are suitable for operation at the time of diagnosis, which means chemotherapy has been the major treatment for most of the pancreatic adenocarcinoma patients [3]. Gemcitabine (2'-deoxy-2'-difluorodeoxycytidine), a nucleoside analog, has been confirmed to be the first effective drug in pancreatic adenocarcinoma treatment by inhibiting DNA synthesis and stimulating apoptosis of cancer cells [4]. The gemcitabine-related therapy is the medical treatment scanty of clinically effects for pancreatic adenocarcinoma. In addition, only less than $20 \%$ pancreatic adenocarcinoma patients are sensitive to gemcitabine treatment, remaining the major challenge for pancreatic adenocarcinoma treatments [5]. Thus, it will contribute to the development of a novel therapeutic strategy to explore the mechanisms underlying gemcitabine resistance and enhance the efficacy of gemcitabine in pancreatic adenocarcinoma treatment [6].

P70S6K1, one of the most important downstream targets of mTOR, can be activated by the PI3K/PTEN/ AKT signaling pathway and functions as a key regulator in various cellular functions such as cell cycle, cell apoptosis and chemoresistance [7]. Given the significant role of p70S6K1 in cellular functions, p70S6K1 is proven to be the multifunctional hallmark in cancer therapy [8]. In addition, recent studies demonstrated that p70S6K1 is involved in nucleotide synthesis via regulating enzymatic 
activities of carbamoyl phosphate synthetase 2 (CAD) $[9,10]$, indicating the potential role of p70S6K1 in gemcitabine action.

MicroRNAs are small non-coding regulatory RNAs that have been confirmed to participate in human tumorigenesis by directly targeting tumor related genes $[11,12]$. Recent studies in pancreatic adenocarcinoma show indispensable roles of miRNAs in various cellular functions, for example, miRNA-21, miRNA-33a, miRNA-155 and miRNA-218 exhibit important roles in tumor proliferation, invasion, metastasis, and apoptosis [13]. However, only a few miRNAs were identified to be involved in gemcitabine chemoresistance, such as miR-21, miR-181b and miR-17-92 cluster [14-16]. MiRNA-145 has been known as a tumor suppressor which is frequently downregulated in various types of cancer including breast cancer [17], colon cancer [18], prostate cancer [19], bladder cancer [20], and osteosarcomas [21]. Although some studies indicate the oncogenic potential of miR-145 in SW620 cells showing increasing cell proliferation/metabolic activity, miRNA-145 participates in cancer development mostly by targeting significant oncogenes and effectively suppressing their expression in different signal pathways, thus inhibiting cancer cell proliferation, invasion and metastasis or enhancing chemosensitivity [22, 23]. Characterization of global microRNA expression reveals anticancer potential of miR145 in metastatic colorectal cancer. Early evidence from our lab demonstrated that miR-145 negatively regulated p70S6K1 expression at the posttranscriptional level in colon cancer [18]. Here we demonstrate that miR-145 increases sensitivity of pancreatic adenocarcinoma cells to gemcitabine treatment, providing new insights into the role of miR-145/P70S6K1 in mediating gemcitabine chemosensitivity.

\section{RESULTS}

\section{Gemcitabine treatment induces miR-145 up- regulation in pancreatic adenocarcinoma cells}

To identify miRNAs whose expression levels were altered in pancreatic cells in response to gemcitabine treatment, we used CCK8 assay to test the effective concentrations of gemcitabine treatment in Bxpc-3 and Panc-1 cells, and found out that Bxpc-3 was sensitive, whereas Panc-1 was resistant to gemcitabine treatment, with more than 100-fold higher IC50 in Panc-1 cells (Figure 1A and 1B). After determining the appropriate gemcitabine concentrations in these cell lines, we treated Bxpc-3 cells with gemcitabine $(2.5 \mu \mathrm{M})$ or vehicle control, and quantified miRNA expression profile by using qRTPCR analysis. We observed that miR-145 was the most significantly up-regulated miRNA upon treatment (Figure 1C), thus we selected miR-145 as a candidate miRNA in tumor progression and chemotherapy for further study. When Bxpc-3 and Panc-1 cells were exposed to gemcitabine treatment at different concentrations, expression levels of miR-145 were increased in a dosedependent manner in Bxpc-3 cells, whereas there was no significant change on miR-145 expression in Panc-1 cells (Figure 1D and 1E), suggesting that miR-145 expression was substantially up-regulated by gemcitabine treatment in gemcitabine-sensitive cells and the block of miR-145 enhancement in gemcitabine-resistant cells may be a potential mechanism for gemcitabine chemoresistance.

\section{MiR-145 is involved in cell migration and gemcitabine chemosensitivity in pancreatic adenocarcinoma cells}

Stable cell lines over-expressing miR-145 or negative control miRNA (miR-NC) were established using lentivirus carrying miR-145 or miR-NC, and verified by detecting the expression of miR-145 levels in Bxpc-3 and Panc-1 cells (Figure 2A). One of the key characteristics of malignant tumor is migration, we next investigated the effects of miR-145 over-expression on migration in vitro. Forced expression of miR-145 dramatically inhibited 65\% and $50 \%$ cell migration capacity of Bxpc-3 and Panc-1 cells, respectively (Figure $2 \mathrm{~B}$ and $2 \mathrm{C}$ ). The miR-145overexpressing pancreatic adenocarcinoma cells were used to analyze cell viability upon gemcitabine treatment. The results showed that over-expression of miR-145 in pancreatic adenocarcinoma cells significantly increased the sensitivity to gemcitabine treatment (Figure 2D and $2 \mathrm{E})$. To examine the basal levels of miR-145 expression in these cell lines, we found that compared with Panc-1, Bxpc3 has higher expression levels of miR-145. Therefore, we knocked down miR-145 in Bxpc-3 cells to further verify the biological function of miR-145. We found that miR-145 interference effectively increased the viability of Bxpc-3 cells upon gemcitabine treatment (Figure 2F), and cell proliferation was enhanced by miR-145 inhibition under the treatment of gemcitabine in Bxpc-3 cells (Figure $2 \mathrm{G})$. Furthermore, Transwell assay showed that miR-145 interference enhanced the migration of Bxpc-3 cells.

\section{P70S6K1 is a direct target of miR-145}

Growing evidence indicates that p70S6K1 pathway is involved in cell growth, metastasis and drug resistance. Bioinformatics program was used to predict targets of miR-145, among which p70S6K1 was one of the putative targets of miR-145. Next, we examined whether p70S6K1 is a direct target of miR-145. The sequences of p70S6K1 3'-UTR region with miR-145 potential binding site was shown in Figure 3A. To verify whether miR-145 directly targets p70S6K1, p70S6K1 3'-UTR sequences containing putative binding sites of wild type (WT) or the mutant one (mut) (Figure 3A) were cloned into pMIR-REPORTER vector. HEK293T cells were co-transfected with the wild 
A

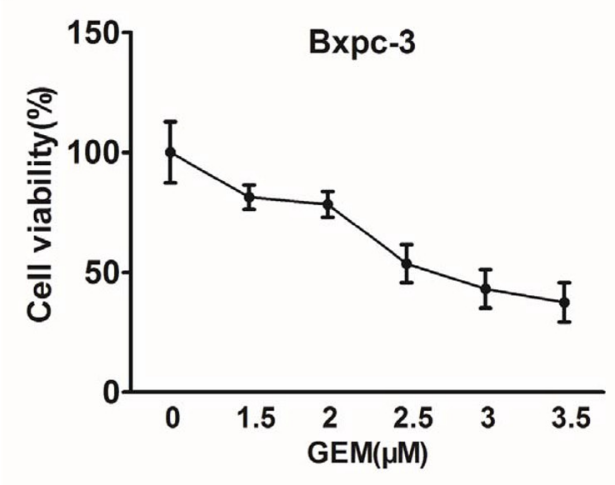

B

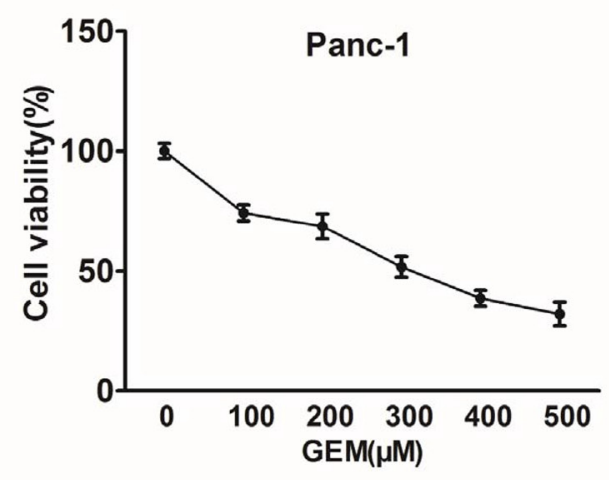

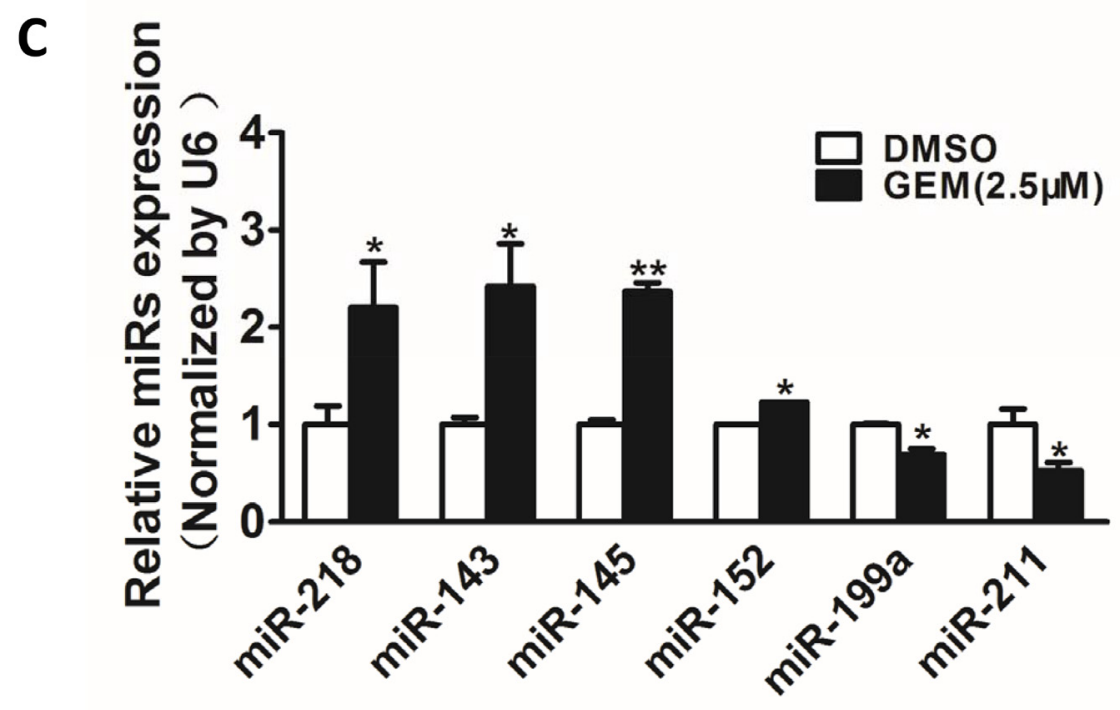
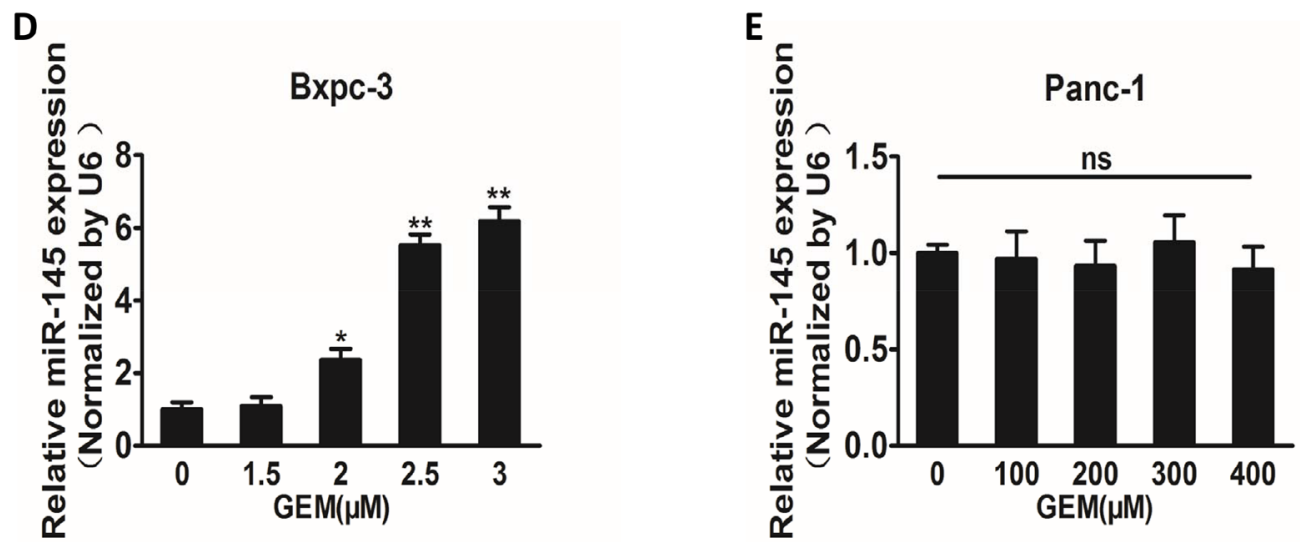

Figure 1: Gemcitabine treatment induces miR-145 up-regulation in gemcitabine-sensitive pancreatic adenocarcinoma cells. A and B. Pancreatic adenocarcinoma cells Bxpc-3 (A) and Panc-1 (B) were treated with gemcitabine (GEM) for $48 \mathrm{~h}$ at different doses. The cell viability was analyzed by CCK-8 assay, and normalized to cells without gemcitabine treatment. C. Expression levels of miRNAs in Bxpc-3 cells were analyzed by RT-qPCR analysis with the treatment of gemcitabine $(2.5 \mu \mathrm{M})$ for $48 \mathrm{~h}$. D, E. Expression levels of miR-145 in Bxpc-3 (D) and Panc-1 (E) cells treated with different doses of gemcitabine. Data from three independent experiments were shown as mean \pm SEM. * indicated significant difference at $\mathrm{P}<0.05$. ;** indicated significant difference at $\mathrm{P}<0.01$. 


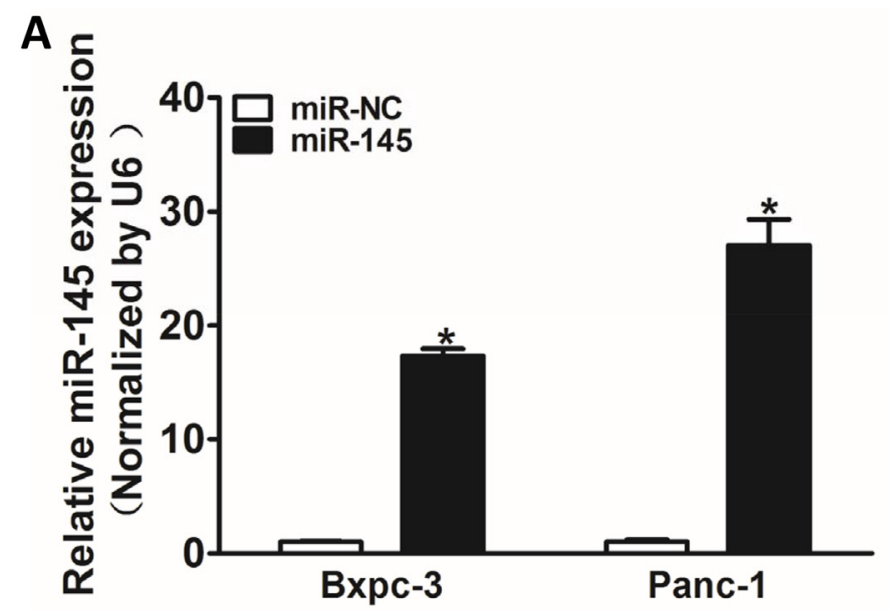

B

Bxpc-3
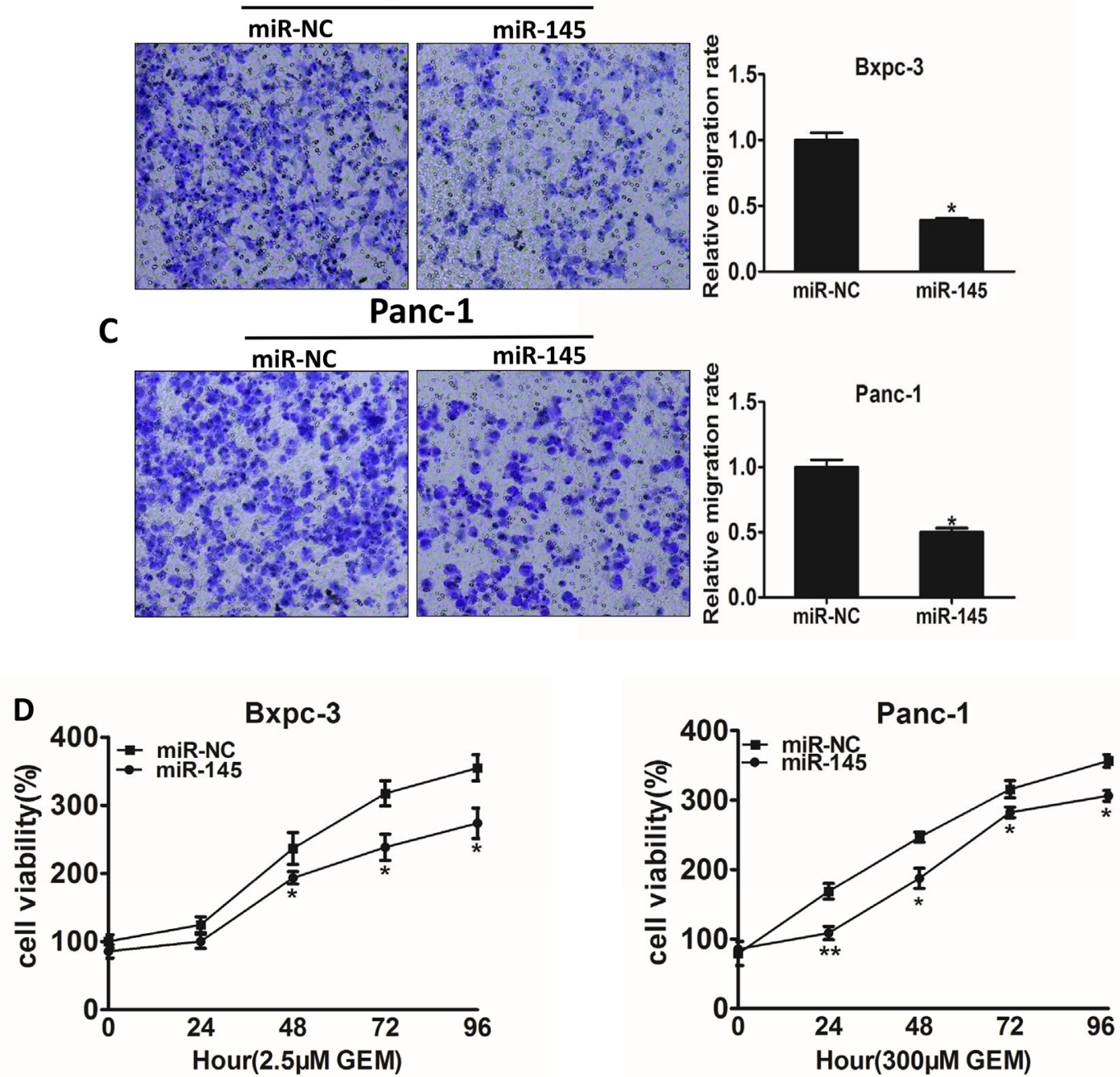

Figure 2: MiR-145 over-expression attenuates cell migration and enhances gemcitabine chemosensitivity in pancreatic adenocarcinoma cells. A. The levels of miR-145 were detected using RT-qPCR in the two cell lines over-expressing miR-145 or miRNA negative control (miR_NC). B, C. Transwell assay was used to measure cell migration in both Bxpc-3 (B) and Panc-1 (C) cells stably expressing negative control miRNA (miR-NC) or miR-145 precursor, and the results were normalized to cells expressing miR-NC. D. The effects of miR-145 over-expression on cell viability in Bxpc-3 and Panc-1 cells treated with indicated concentrations of gemcitabine were analyzed using CCK-8 assay.

(Continued) 


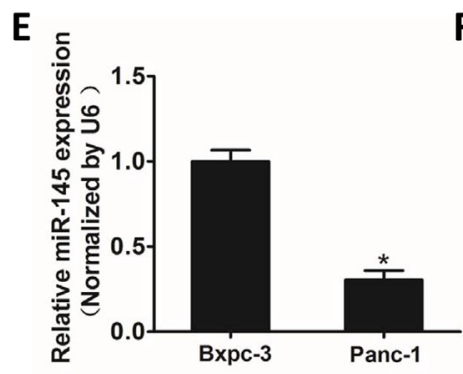

$\mathbf{F}$

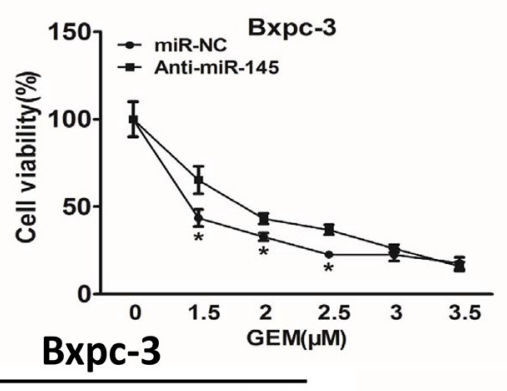

G

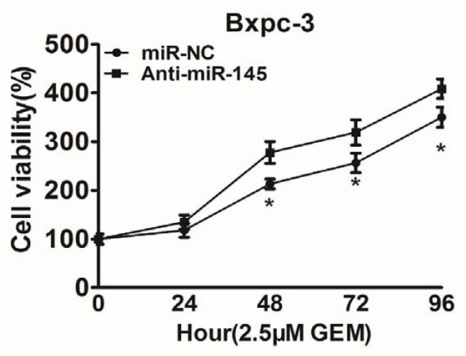

H
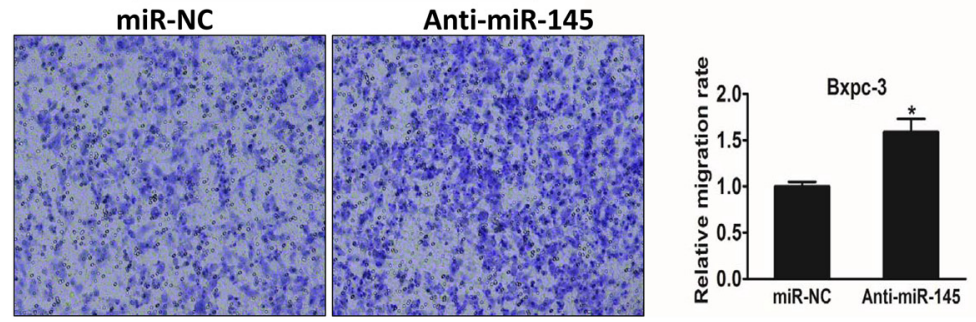

Figure 2 (Continued): E. The endogenous expression levels of miR-145 in Bxpc-3 and Panc-1 cells. F. Bxpc-3 and Panc-1 transfected with miR-145 inhibitor (Anti-miR-145) or the negative control miRNA inhibitor (miR-NC) were treated with different concentration of gemcitabine for $48 \mathrm{~h}$. The cell viability was analyzed using CCK-8 assay. G. CCK-8 assay was used to analyze the cell viability of Bxpc-3 cells transfected with miR-145 inhibitor or miR-NC for $48 \mathrm{~h}$ at indicated time points. H. Cell migration of Bxpc-3 cells transfected with miR-145 inhibitor. Data represent mean \pm SEM from 3 replicates. * indicated significant difference at $\mathrm{P}<0.05$; ** indicated significant difference at $\mathrm{P}<0.01$.

type (WT) or mutant (Mut) p70S6K1 3'-UTR luciferase reporter vector together with miR-145 mimics or miR$\mathrm{NC}$ for $24 \mathrm{~h}$, and luciferase activities in those cells were measured. Overexpression of miR-145 significantly inhibited the luciferase activities of wild-type, but not mutant p70S6K1 3'-UTR reporter in HER293T cells (Figure 3B), suggesting that miR-145 targets p70S6K1 through the binding to the seed sequence of its 3'-UTR. To determine whether p70S6K1 was down-regulated under gemcitabine treatment, Bxpc-3 cells were treated with gemcitabine for $48 \mathrm{~h}$ and the protein levels of p70S6K1, $\mathrm{p}$-S6, HIF- $1 \alpha$ and GAPDH were examined. We found that the levels of p70S6K1, p-S6 and HIF-1 $\alpha$ were significantly suppressed in a dose-dependent manner (Figure 3C). In addition, miR-145 over-expression markedly suppressed protein expression levels of p70S6K1, p-S6, HIF$1 \alpha$ and VEGF in pancreatic cancer cells (Figure 3D), confirming that miR-145 targets p70S6K1 and inhibits relative signaling molecules in these cells. Consistent with HIF- $1 \alpha$ and VEGF protein down-regulation in miR145-overexpressing Bxpc-3 and Panc-1 cells, forced expression also suppressed VEGF mRNA levels in these cells (Figure 3E). Furthermore, as shown in Figure 3F, we measured mRNA levels of miR-145 and p70S6K1 in pancreatic adenocarcinoma tumor tissues and determined the correlation between p70S6K1 levels and miR-145 expression levels (Figure 3F). Spearman's rank correlation analysis showed that expression levels of p70S6K1 and
miR-145 in pancreatic adenocarcinoma specimens were inversely correlated (Spearman's correlation $r=-0.5361$ ).

\section{Overexpression of $\mathrm{p} 70 \mathrm{~S} 6 \mathrm{~K} 1$ reverses the inhibitory effect of miR-145 on cell migration and chemoresistance}

To identify potential molecular mechanism of miR-145 in pancreatic adenocarcinoma development and treatment resistance, we determined whether miR-145 inhibited cell migration and cell viability under gemcitabine treatment though p70S6K1. The overexpression of miR-145 decreased endogenous p70S6K1 protein levels, whereas forced expression of p70S6K1 without 3'-UTR dramatically increased p70S6K1 expression levels (Figure 4A). Over-expression of p70S6K1 rescued the ability of cell migration which was suppressed by miR-145 over-expression in pancreatic adenocarcinoma cell lines (Figure 4B and $4 \mathrm{C})$. Furthermore, cell growth rate in the presence of gemcitabine was assayed by CCK- 8 proliferation assay at different time points. Our results showed that the restoration of p70S6K1 expression in the cells stably overexpressing miR-145 significantly increased gemcitabine chemoresistance (Figure 4D). To further study whether p70S6K1 is required for gemcitabine-resistance in pancreatic cancer cells, we confirmed that knockdown of p70S6K1 using its shRNA significantly increased cell 


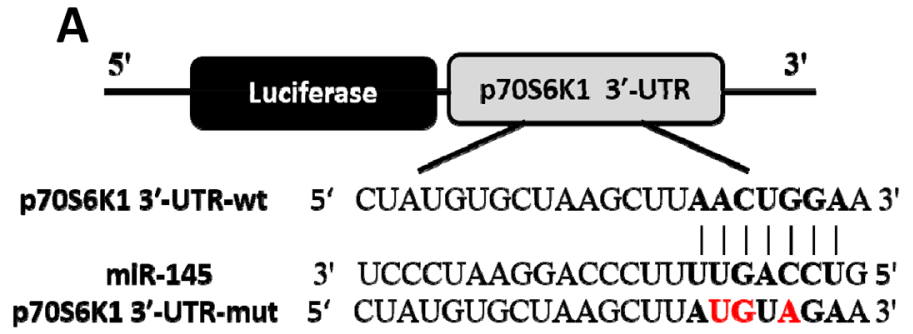

B

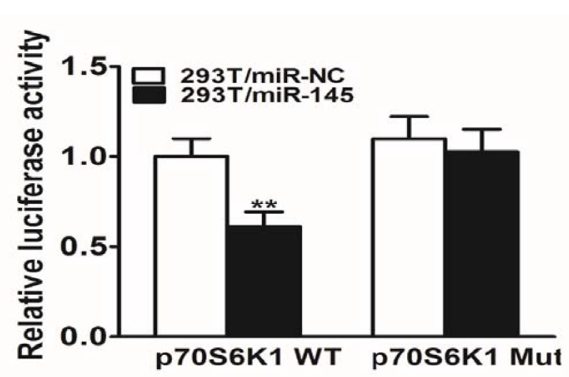

C
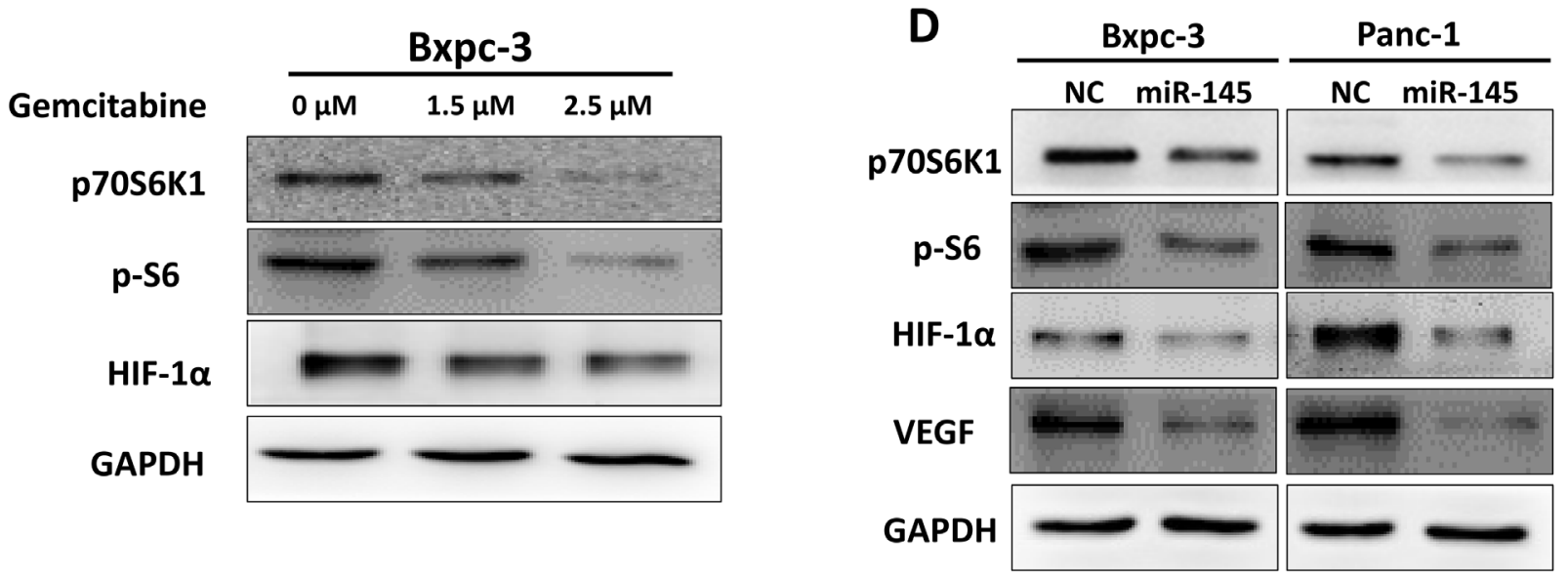

$\mathbf{E}$

$\mathbf{F}$
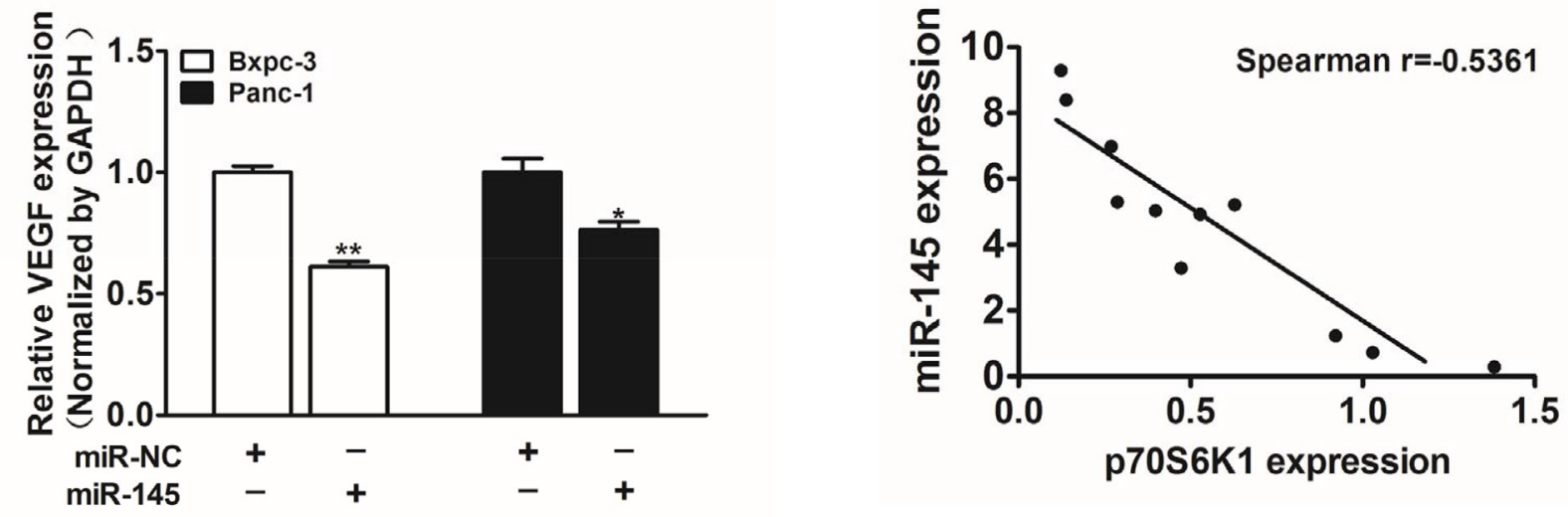

Figure 3: P70S6K1 is a direct target of miR-145. A. Schematic diagram of putative miR-145 binding site in the $3^{\prime}-\mathrm{UTR}$ region of p70S6K1 in human. The seed sequence of miR-145 matches 3'-UTR regions of p70S6K1 (in bold). The mutated nucleotides of the p70S6K1 3'-UTR were labeled in red. B. HEK-293T cells were co-transfected with control (miR-NC) or pre-miR-145 mimics along with WT or mutant p70S6K1 luciferase reporter as indicated. Luciferase activities were measured $24 \mathrm{~h}$ post transfection using the dual-luciferase reporter assay system. C. Cells were treated with different doses of gemcitabine (0, 1.5 and $2.5 \mu \mathrm{M})$, the expression levels of p70S6K1, p-S6 and HIF-1 $\alpha$ were determined by Western blotting. GAPDH is used as an internal control. D. Cells were transfected with miR-145 or miR-NC. After $72 \mathrm{~h}$, the expression levels of p70S6K1, p-S6, HIF-1 $\alpha$ and VEGF were determined as above. E. VEGF levels were measured by RT-qPCR in cell lines stably overexpressing miR-NC and miR-145, and normalized to level of GAPDH. F: Spearman's correlation analysis was used to detect the correlation between expression levels of p70S6K1 and miR-145 at mRNA level in human pancreatic tumor specimens. Data represent mean \pm SEM of three replicates. * indicated significant difference at $\mathrm{P}<0.05$; ** indicated significant difference at $\mathrm{P}<0.01$. 
A

Bxpc-3

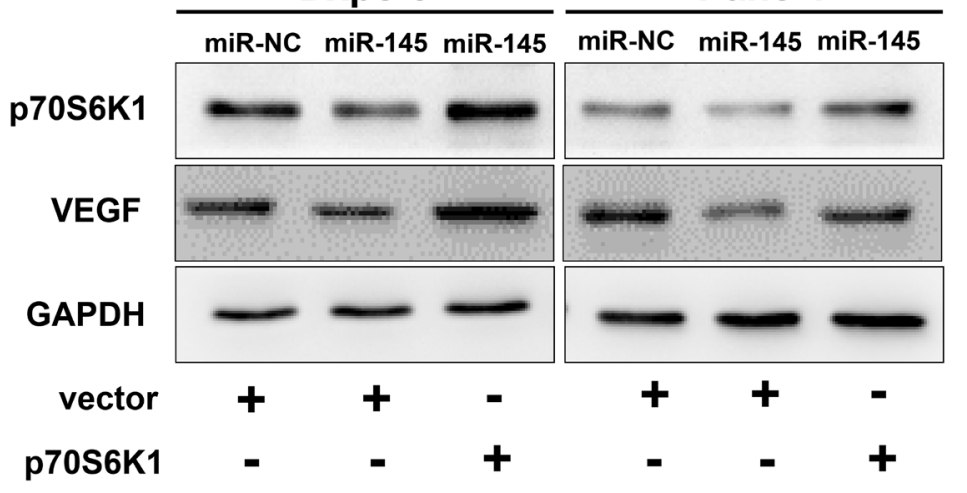

8

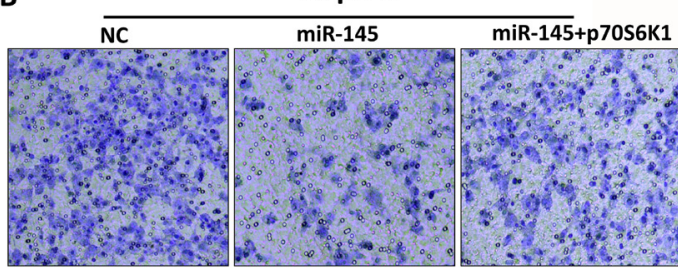

c

Panc-1

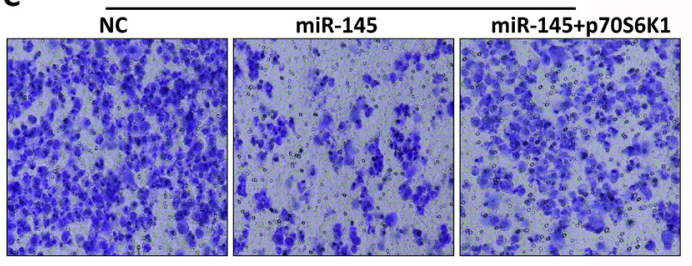

D

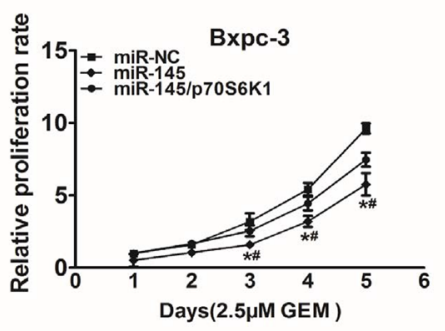

$\mathbf{E}$

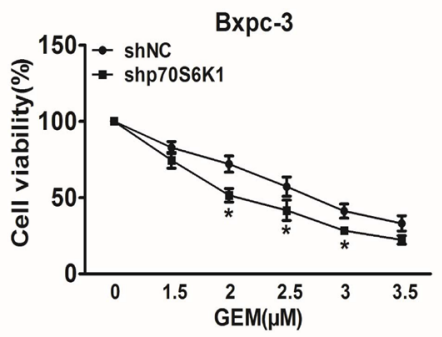

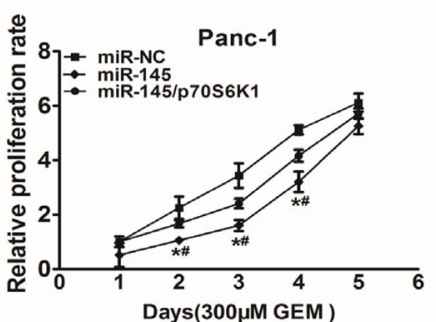

Panc-1

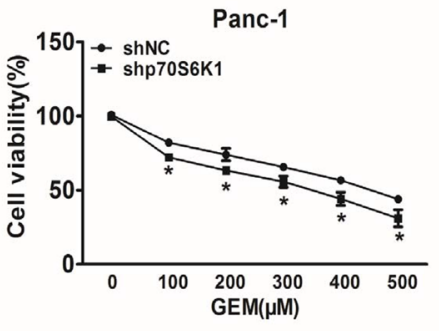

Figure 4: Over-expression of p70S6K1 reverses the inhibitory effects of miR-145. A. Bxpc-3 and Panc-1 cells stably expressing miR-NC or miR-145 were transfected with p70S6K1 cDNA construct without 3'-UTR or vector control. After $72 \mathrm{~h}, \mathrm{p} 70 \mathrm{~S} 6 \mathrm{~K} 1$ and GAPDH expression levels were measured using immunoblotting assay as above. B, C. The effects of p70S6K1 forced expression on cell migration were analyzed using Transwell assay in Bxpc-3 (B) and Panc-1 (C) cells stably expressing miR-NC or miR-145. D. The cells above were treated with indicated concentrations of gemcitabine at different time point, cell proliferation was analyzed using CCK8 Assay Kit and normalized to the number of miR-NC group without gemcitabine treatment. E. Bxpc-3 and Panc-1 cells were transduced with lentivirus carrying shRNA against p70S6K1 or negative control (shNC) and selected with puromycin. The stable cells were treated with different doses of gemcitabine and cell viability was determined as above. Data represent mean \pm SEM. of three replicates. *indicates significant difference compared to control at $\mathrm{P}<0.05$; \#indicates significant difference compared to miR-145 treatment alone at $\mathrm{P}<0.05$. 
viability upon gemcitabine treatment in both cell lines (Figure 4E).

\section{MiR-145 inhibits VEGF transcriptional activation through targeting p70S6K1 in pancreatic adenocarcinoma cells}

Previous studies have shown the importance of p70S6K1/HIF-1 $\alpha$ /VEGF pathway in the regulation of angiogenesis and tumor progression. It has been reported that HIF- $1 \alpha$ activates the expression of VEGF gene by binding to the hypoxia response element (HRE) in the VEGF promoter region. To determine whether miR145 attenuates VEGF transcriptional activation through targeting p70S6K1, we analyzed the effect of miR145 on the wild type VEGF promoter reporter plasmid (pMAP11WT) containing the HIF-1 $\alpha$ binding site or the mutant plasmid (pMAP11Mut) with or without p70S6K1 over-expression. As shown in Figure 5A and 5B, forced expression of p70S6K1 restored miR-145-inhibited the wild type VEGF reporter activity in Bxpc-3 and Panc1 cells, but did not affect the mutant reporter activity, indicating that miR-145 inhibits VEGF transcriptional activation by targeting p70S6K1.

\section{DISCUSSION}

Pancreatic adenocarcinoma is one of the most common digestive tract malignant tumors, which is difficult in early diagnosis and treatment. In present, distant metastasis, relapse, and drug resistance are still very difficult problems in cancer therapy. Gemcitabine, a

A

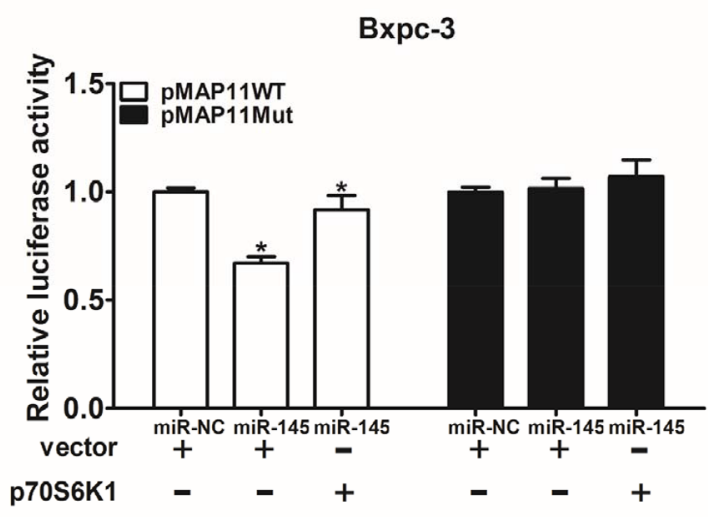

first-line treatment drug of pancreatic adenocarcinoma, is widely used in clinical practice. Currently, some literatures suggest that miRNAs can affect drug sensitivity of tumor cells to gemcitabine treatment, including miR-218, miR$33 \mathrm{a}$ and miR-223 [24-26], but the role and molecular mechanism of miRNAs in pancreatic adenocarcinoma cells to gemcitabine treatment have not been reported. We found that compared with Bxpc-3, a much higher concentration of gemcitabine was required to inhibit the cell viability of Panc-1, confirming that Panc-1 is a gemcitabine-resistant cell line. We also found that gemcitabine could up-regulate expression levels of miR-145 in Bxpc-3, the gemcitabinesensitive cells, but not in Panc-1 cells, suggesting specific role of miR-145 in gemcitabine chemosensitivity in pancreatic adenocarcinoma cells.

MiR-145 is known as a tumor suppressor, which plays an important role in tumor development. The expression levels of miR-145 are found to be downregulated in many types of cancers, including pancreatic adenocarcinoma [27-31]. The expression profiles and biological role of miR-145 in pancreatic adenocarcinoma has been reported [31,32], but the role and mechanisms of miR-145 in regulating drug resistance remain to be elucidated. In this study, we found that in both gemcitabine-sensitive cells Bxpc-3 and gemcitabineresistant cells Panc-1, overexpression of miR-145 significantly inhibited cell migration by directly targeting p70S6K1. Moreover, overexpression of miR-145 increased chemosensitivity of cancer cells to gemcitabine treatment in vitro, indicated by decreased cell viability. Furthermore, p70S6K1 is a direct target of miR-145 in pancreatic cancer cells. Forced expression of p70S6K1
B

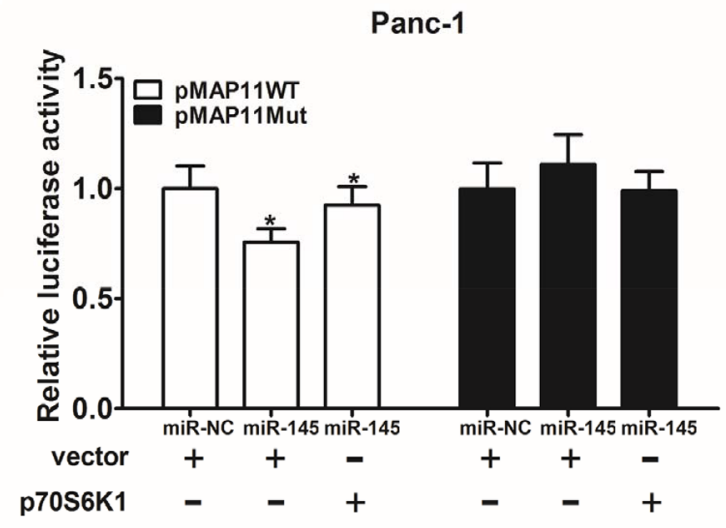

Figure 5: MiR-145 inhibits VEGF transcriptional activation through targeting p70S6K1 in pancreatic adenocarcinoma cells. A. Bxpc-3 cells were co-transfected with wild-type VEGF reporter (pMAP11-WT) or mutant VEGF reporter (pMAP11-mut), miRNC negative control or miR-145 precursor, p70S6K1 cDNA or vector control, and pRL-TK plasmid. The luciferase activities were measured using firefly and Renilla dual luciferase reporter assay, and normalized to those of the control. B. The similar experiment was performed using PANC-1 cells. Data represent mean \pm SEM. from three replicates. * indicated significant difference at $\mathrm{P}<0.05$. 
reversed miR-145-suprressed cell proliferation and drug resistance of pancreatic adenocarcinoma cells, suggesting that p70S6K1 plays a central role in miR-145-induced chemosensitivity to gemcitabine treatment. MiR-143 may have similar effect and regulation mechanism as miR-145 in cells, which would also be interesting to be studied in the future.

The activation of $\mathrm{p} 70 \mathrm{~S} 6 \mathrm{~K} 1$ affects the expression levels of two important angiogenesis-related downstream signaling molecules, HIF-1 $\alpha$ and VEGF, which are involved in tumorigenesis and cancer development [3337]. It has also been demonstrated that HIF- $1 \alpha$ regulates VEGF expression at the transcriptional level by binding to its promoter [38]. In our study, overexpression of miR-145 suppressed the expression levels of HIF-1 $\alpha$ and VEGF, which is consistent with its inhibitory effect on p70S6K1. Furthermore, forced expression of p70S6K1 in miR-145over-expressing pancreatic adenocarcinoma cells restored the expression levels of HIF-1 $\alpha$ and VEGF which were inhibited by miR-145, suggesting that miR-145 controls expression of HIF-1a and VEGF by targeting p70S6K1 in the cells.

In summary, our findings showed that Panc-1 resistance to gemcitabine is due to the down-regulation of miR-145, suggesting that miR-145 may be an indicator for the development of pancreatic adenocarcinoma. Forced expression of miR-145 inhibited the cell viability, migration, HIF-1 $\alpha$, VEGF expression and enhanced the chemosensitivity to gemcitabine through its direct target p70S6K1 in pancreatic adenocarcinoma. These results suggest that miR-145 may be a potential target for adjuvant therapy to enhance the chemosensitivity to gemcitabine in the future. Our findings provided a new molecular basis for the application of miR-145 in pancreatic adenocarcinoma, which may be helpful in developing microRNA-based drug for treating pancreatic adenocarcinoma in the future.

\section{MATERIALS AND METHODS}

\section{Cell culture and reagents}

Human pancreatic adenocarcinoma cell lines, Panc1 and Bxpc-3 were maintained in Dulbecco's Modified Eagle's medium with high glucose and RPMI-1640 medium with low glucose, respectively, and supplemented with $10 \%$ fetal bovine serum and antibiotics (100 units/ $\mathrm{ml}$ penicillin and $100 \mathrm{mg} / \mathrm{ml}$ streptomycin). Cells were incubated in a $5 \% \mathrm{CO} 2$ incubator at $37^{\circ} \mathrm{C}$. Trypsin $(0.25 \%)$ solution was used to detach the cells from the culture flask. P70S6K1 and VEGF antibodies were purchased from Santa Cruz Biotechnology (Santa Cruz, CA, USA). Antibodies against HIF-1 $\alpha$ and GAPDH were purchased from Bioworld Technology (Atlanta, Georgia, USA). Gemcitabine was obtained from Sigma (St. Louise, $\mathrm{MO})$.

\section{Luciferase reporter assays}

The 3'-UTR of p70S6K1 constructs containing predicted miR-145 seed-matching sites from cDNA library were amplified by PCR using Pfu DNA polymerase, then cloned into pMIR-REPORTER vector (Ambion). Primers of wide type (WT) and corresponding mutant sites (Mut) used for reporter constructions were shown in Supplementary Table S1. All the 3'-UTR products were sequenced and verified. For reporter assays, HEK293T cells were transiently cotransfected with WT or Mut plasmid, pRL-TK plasmid and miRNA-145 mimics (GenePharma, Shanghai, China) or miRNA-negative control (miR-NC) using lipofectamine 2000 (Invitrogen). Firefly and renilla luciferase activities were measured $24 \mathrm{~h}$ after transfection using a dual luciferase assay kit (Promega, Shanghai, China). The relative luciferase activities were calculated and normalized to the control.

To detect the effects of miR-145 on transcriptional activation of VEGF, the downstream of p70S6K1, VEGF reporter plasmid pMAP11-WT or pMAP11-Mut was cotransfected into Bxpc-3 and Panc-1 cells with pRL-TK plasmid and miR-145 mimics or miR-NC. Firefly and Renilla luciferase activities were measured $24 \mathrm{~h}$ after the transfection. Experiments were performed in three independent replicates.

\section{RNA extractions, reverse transcription PCR and quantitative real time- PCR}

Total RNAs were extracted from cells with TRIzol (Invitrogen, CA, USA). The stem-loop RT-PCR assay was performed to quantify the miRNAs expression levels using the HiScript RT Reagent Kit (Vazyme Biotech, Nanjing, China) according to the manufacturer's instructions. To determine the mRNA levels of VEGF, total RNAs were reversely transcribed using HiScript RT Reagent Kit (Vazyme Biotech, Nanjing, China). RT-qPCR was performed to detect mRNA expression levels using the SYBR Premix DimerEraser (Takara, Dalian, China) on a 7900 HT system. The expression levels of U6 or GAPDH were used as internal control. The expression levels of miRNAs in each group were calculated by relative quantification $(2-\Delta \Delta \mathrm{Ct})$. The primers used in this study are shown in Supplementary Table S1.

\section{Cell proliferation}

To detect the gemcitabine effects on the pancreatic adenocarcinoma cell viability, cells were seeded into 96-well plates at 2000 per well. After cellular adhesion, medium containing gemcitabine at different concentrations was added to the cells. Cell viability was assessed using the CCK8 Assay (CCK8 kit, Dojindo Laboratories, Kumamoto, Japan) $48 \mathrm{~h}$ after the transfection. CCK8 reagents were prepared in fresh medium $(100 \mu \mathrm{l}$ medium containing $10 \mu \mathrm{l}$ CCK8 solutions) and applied to the cells. 
The absorbance at $450 \mathrm{~nm}$ for each well was analyzed on a spectrophotometer.

To determine the effects of miR-145 on cell growth, Bxpc-3 and Panc- 1 cells were seeded in a 6-well plate and cultured overnight. After transient transfection with miR145 and miR-NC precursors, the cells were trypsinized and seeded into a 96-well plate. CCK8 Assay was used to measure cell proliferation at different indicated time points.

\section{Cell migration assay}

Transwell chambers (24-well insert; Corning, NY, USA) were used to analyze cell migration in accordance with the manufacturer's instructions. Cells were seeded in the upper well of the chamber in DMEM or RPMI-1640 medium without serum, while the lower chamber well contained DMEM or RPMI-1640 medium supplemented with $10 \%$ FBS to stimulate cell migration. After incubation for $12 \mathrm{~h}$, cells on the upper membrane surface were removed while the bottom cells were fixed with $3 \%$ paraformaldehyde, stained with $0.1 \%$ crystal violet, and 5-6 random fields were collected for quantification for each well. The cells were finally extracted with $33 \%$ acetic acid and detected quantitatively using a standard microplate reader (at $570 \mathrm{~nm}$ ). Three independent experiments were performed for each experimental group.

\section{Establishment of stable cell lines expressing miR-145}

Lentivirus carrying miR-145 and miR-NC were packaged using lentiviral packaging kit in HEK-293T cells according to the manufacturer's instructions (Open Biosystems, AL, USA). Stable Bxpc-3 and Panc-1 cells over-expressing miR-145 or miR-NC were generated by the infection of lentivirus (Open Biosystems), followed by puromycin (Life Technologies) selection.

\section{Western blotting}

Cells were lysed with RIPA buffer $(150 \mathrm{mM} \mathrm{NaCl}$, $100 \mathrm{mM}$ Tris, $\mathrm{pH} 8.0,0.1 \%$ SDS, $1 \%$ Triton $\mathrm{X}-100$, $1 \%$ sodium deoxycholate, $5 \mathrm{mM}$ EDTA, and $10 \mathrm{mM}$ $\mathrm{NaF}$ ) supplemented with $1 \mathrm{mM}$ sodium vanadate, $2 \mathrm{mM}$ leupeptin, $2 \mathrm{mM}$ aprotinin, $1 \mathrm{mM}$ phenylmethylsulfonyl fluoride (PMSF), $1 \mathrm{mM}$ DTT, and $2 \mathrm{mM}$ pepstatin. Total proteins were collected by centrifugation at $12,000 \mathrm{rpm} 4^{\circ} \mathrm{C}$ for $15 \mathrm{~min}$. After quantification, 20 $\mu \mathrm{g}$ protein lysates were separated by SDS-PAGE and subsequently transferred to polyvinylidene difluoride membranes (Millipore, Billerica, MA, USA), membranes were blocked with 5\% nonfat dry milk for $2 \mathrm{~h}$ and incubated with primary antibodies. Protein bands were detected by incubation with horseradish peroxidase-conjugated antibodies and visualized with an electrochemiluminescence detection system (Thermo Scientific).

\section{Statistical analysis}

Values were obtained from at least three independent experiments and presented as means \pm SEM. Comparison between two groups was conducted using the t-test, while comparisons between multiple groups of data were performed using analysis of variance (ANOVA). It was considered to be statistically significant at $\mathrm{P}<0.05$.

\section{ACKNOWLEDGMENTS}

This work was supported in part by National Natural Science Foundation of China (81472944, 81320108019, 81302182); by National Institutes of Health grants (R01ES020868 and R01CA193511); and by American Cancer Society (RSG NEC -129306).

\section{CONFLICTS OF INTEREST}

The authors confirm that this article content has no conflicts of interest.

\section{REFERENCES}

1. Jemal A, Siegel R, Xu J, Ward E. Cancer statistics, 2010. CA Cancer J Clin. 2010; 60:277-300.

2. Siegel R, Naishadham D, Jemal A. Cancer statistics, 2013. CA Cancer J Clin. 2013; 63:11-30.

3. Vincent A, Herman J, Schulick R, Hruban RH, Goggins M. Pancreatic cancer. Lancet. 2011; 378:607-620.

4. Plunkett W, Huang P, Searcy CE, Gandhi V. Gemcitabine: preclinical pharmacology and mechanisms of action. Seminars in oncology. 1996; 23:3-15.

5. Zhang S, Zhang XQ, Huang SL, Chen M, Shen SS, Ding XW, Lv Y, Zou XP. The Effects of HSP27 on GemcitabineResistant Pancreatic Cancer Cell Line Through Snail. Pancreas. 2015; 44:1121-1129.

6. Bhattacharjee V, Zhou Y, Yen TJ. A synthetic lethal screen identifies the Vitamin D receptor as a novel gemcitabine sensitizer in pancreatic cancer cells. Cell cycle. 2014; 13:3839-3856.

7. Bjornsti MA, Houghton PJ. The TOR pathway: a target for cancer therapy. Nature reviews Cancer. 2004; 4:335-348.

8. Jiang BH, Liu LZ. Role of mTOR in anticancer drug resistance: perspectives for improved drug treatment. Drug resistance updates: reviews and commentaries in antimicrobial and anticancer chemotherapy. 2008; 11:63-76.

9. Ben-Sahra I, Howell JJ, Asara JM, Manning BD. Stimulation of de novo pyrimidine synthesis by growth signaling through mTOR and S6K1. Science. 2013; 339:1323-1328. 
10. Robitaille AM, Christen S, Shimobayashi M, Cornu M, Fava LL, Moes S, Prescianotto-Baschong C, Sauer U, Jenoe P, Hall MN. Quantitative phosphoproteomics reveal mTORC1 activates de novo pyrimidine synthesis. Science. 2013; 339:1320-1323.

11. Ambros V, Lee RC. Identification of microRNAs and other tiny noncoding RNAs by cDNA cloning. Methods in molecular biology. 2004; 265:131-158.

12. Pillai RS, Bhattacharyya SN, Filipowicz W. Repression of protein synthesis by miRNAs: how many mechanisms? Trends in cell biology. 2007; 17:118-126.

13. Brunetti O, Russo A, Scarpa A, Santini D, Reni M, Bittoni A, Azzariti A, Aprile G, Delcuratolo S, Signorile M, Gnoni A, Palermo L, Lorusso V, Cascinu S, Silvestris N. MicroRNA in pancreatic adenocarcinoma: predictive/ prognostic biomarkers or therapeutic targets? Oncotarget. 2015; 6:23323-23341. doi: 10.18632/oncotarget.4492.

14. Giovannetti E, Funel N, Peters GJ, Del Chiaro M, Erozenci LA, Vasile E, Leon LG, Pollina LE, Groen A, Falcone A, Danesi R, Campani D, Verheul HM, Boggi U. MicroRNA-21 in pancreatic cancer: correlation with clinical outcome and pharmacologic aspects underlying its role in the modulation of gemcitabine activity. Cancer research. 2010; 70:4528-4538.

15. Takiuchi D, Eguchi H, Nagano H, Iwagami Y, Tomimaru Y, Wada H, Kawamoto K, Kobayashi S, Marubashi S, Tanemura M, Mori M, Doki Y. Involvement of microRNA$181 \mathrm{~b}$ in the gemcitabine resistance of pancreatic cancer cells. Pancreatology. 2013; 13:517-523.

16. Cioffi M, Trabulo SM, Sanchez-Ripoll Y, Miranda-Lorenzo I, Lonardo E, Dorado J, Reis Vieira C, Ramirez JC, Hidalgo M, Aicher A, Hahn S, Sainz B, Jr., Heeschen C. The miR17-92 cluster counteracts quiescence and chemoresistance in a distinct subpopulation of pancreatic cancer stem cells. Gut. 2015; 64:1936-1948.

17. Spizzo R, Nicoloso MS, Lupini L, Lu Y, Fogarty J, Rossi S, Zagatti B, Fabbri M, Veronese A, Liu X, Davuluri R, Croce CM, Mills G, Negrini M, Calin GA. miR-145 participates with TP53 in a death-promoting regulatory loop and targets estrogen receptor-alpha in human breast cancer cells. Cell death and differentiation. 2010; 17:246-254.

18. Xu Q, Liu LZ, Qian X, Chen Q, Jiang Y, Li D, Lai L, Jiang $\mathrm{BH}$. MiR-145 directly targets p70S6K1 in cancer cells to inhibit tumor growth and angiogenesis. Nucleic acids research. 2012; 40:761-774.

19. Wang C, Tao W, Ni S, Chen Q, Zhao Z, Ma L, Fu Y, Jiao Z. Tumor-suppressive microRNA-145 induces growth arrest by targeting SENP1 in human prostate cancer cells. Cancer science. 2015; 106:375-382.

20. Zhu Z, Xu T, Wang L, Wang X, Zhong S, Xu C, Shen Z. MicroRNA-145 directly targets the insulin-like growth factor receptor I in human bladder cancer cells. FEBS letters. 2014; 588:3180-3185.
21. Li E, Zhang J, Yuan T, Ma B. MiR-145 inhibits osteosarcoma cells proliferation and invasion by targeting ROCK1. Tumour biology. 2014; 35:7645-7650.

22. Arndt GM, Dossey L, Cullen LM, Lai A, Druker R, Eisbacher M, Zhang C, Tran N, Fan H, Retzlaff K, Bittner A, Raponi M. Characterization of global microRNA expression reveals oncogenic potential of miR-145 in metastatic colorectal cancer. BMC cancer. 2009; 9:374.

23. Sachdeva M, Zhu S, Wu F, Wu H, Walia V, Kumar S, Elble R, Watabe K, Mo YY. p53 represses c-Myc through induction of the tumor suppressor miR-145. Proceedings of the National Academy of Sciences of the United States of America. 2009; 106:3207-3212.

24. Liu Z, Du R, Long J, Guo K, Ge C, Bi S, Xu Y. microRNA-218 promotes gemcitabine sensitivity in human pancreatic cancer cells by regulating HMGB1 expression. Chinese journal of cancer research [Chinese]. 2015; 27:267-278.

25. Ma J, Fang B, Zeng F, Ma C, Pang H, Cheng L, Shi Y, Wang H, Yin B, Xia J, Wang Z. Down-regulation of miR-223 reverses epithelial-mesenchymal transition in gemcitabineresistant pancreatic cancer cells. Oncotarget. 2015; 6:17401749. doi: 10.18632/oncotarget. 2714.

26. Liang C, Wang Z, Li YY, Yu BH, Zhang F, Li HY. miR33a suppresses the nuclear translocation of beta-catenin to enhance gemcitabine sensitivity in human pancreatic cancer cells. Tumour biology. 2015; 36:9395-9403.

27. Michael MZ, SM OC, van Holst Pellekaan NG, Young GP, James RJ. Reduced accumulation of specific microRNAs in colorectal neoplasia. Molecular cancer research. 2003; $1: 882-891$.

28. Iorio MV, Ferracin M, Liu CG, Veronese A, Spizzo R, Sabbioni S, Magri E, Pedriali M, Fabbri M, Campiglio M, Menard S, Palazzo JP, Rosenberg A, Musiani P, Volinia S, Nenci I, et al. MicroRNA gene expression deregulation in human breast cancer. Cancer research. 2005; 65:7065-7070.

29. Ozen M, Creighton CJ, Ozdemir M, Ittmann M. Widespread deregulation of microRNA expression in human prostate cancer. Oncogene. 2008; 27:1788-1793.

30. Lee HK, Bier A, Cazacu S, Finniss S, Xiang C, Twito H, Poisson LM, Mikkelsen T, Slavin S, Jacoby E, Yalon M, Toren A, Rempel SA, Brodie C. MicroRNA-145 is downregulated in glial tumors and regulates glioma cell migration by targeting connective tissue growth factor. PloS one. 2013; 8:e54652.

31. Papaconstantinou IG, Manta A, Gazouli M, Lyberopoulou A, Lykoudis PM, Polymeneas G, Voros D. Expression of microRNAs in patients with pancreatic cancer and its prognostic significance. Pancreas. 2013; 42:67-71.

32. Khan S, Ebeling MC, Zaman MS, Sikander M, Yallapu MM, Chauhan N, Yacoubian AM, Behrman SW, Zafar N, Kumar D, Thompson PA, Jaggi M, Chauhan SC. MicroRNA-145 targets MUC13 and suppresses growth and 
invasion of pancreatic cancer. Oncotarget. 2014; 5:75997609. doi: 10.18632/oncotarget.2281

33. Plate $\mathrm{KH}$, Breier G, Weich HA, Risau W. Vascular endothelial growth factor is a potential tumour angiogenesis factor in human gliomas in vivo. Nature. 1992; 359:845-848.

34. Semenza GL. Defining the role of hypoxia-inducible factor 1 in cancer biology and therapeutics. Oncogene. 2010; 29:625-634.

35. Liu LZ, Hu XW, Xia C, He J, Zhou Q, Shi X, Fang J, Jiang $\mathrm{BH}$. Reactive oxygen species regulate epidermal growth factor-induced vascular endothelial growth factor and hypoxia-inducible factor-1alpha expression through activation of AKT and P70S6K1 in human ovarian cancer cells. Free radical biology \& medicine. 2006; 41:1521-1533.
36. Zhou Q, Liu LZ, Fu B, Hu X, Shi X, Fang J, Jiang BH. Reactive oxygen species regulate insulin-induced VEGF and HIF-1alpha expression through the activation of p70S6K1 in human prostate cancer cells. Carcinogenesis. 2007; 28:28-37.

37. Liu LZ, Fang J, Zhou Q, Hu X, Shi X, Jiang BH. Apigenin inhibits expression of vascular endothelial growth factor and angiogenesis in human lung cancer cells: implication of chemoprevention of lung cancer. Molecular pharmacology. 2005; 68:635-643.

38. Forsythe JA, Jiang BH, Iyer NV, Agani F, Leung SW, Koos RD, Semenza GL. Activation of vascular endothelial growth factor gene transcription by hypoxia-inducible factor 1 . Molecular and cellular biology. 1996; 16:4604-4613. 